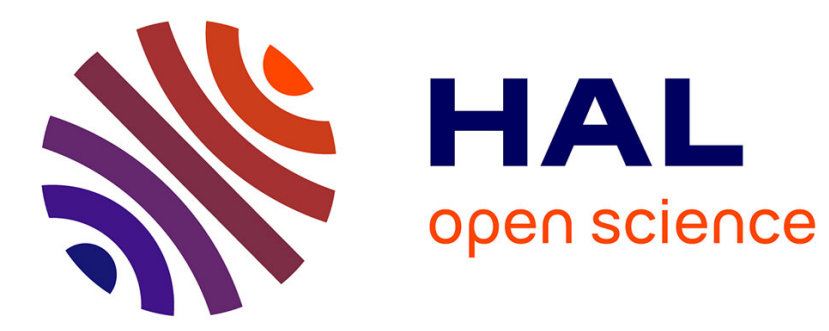

\title{
La préférence pour les comportements prosociaux chez les bébés de 6 mois.
}

Claire Holvoet, Céline Scola, Thomas Arciszewski, Delphine Picard

\section{To cite this version:}

Claire Holvoet, Céline Scola, Thomas Arciszewski, Delphine Picard. La préférence pour les comportements prosociaux chez les bébés de 6 mois.. Enfance, 2017, 4, pp.483-488. hal-01768575

\section{HAL Id: hal-01768575 \\ https://hal-amu.archives-ouvertes.fr/hal-01768575}

Submitted on 25 Apr 2018

HAL is a multi-disciplinary open access archive for the deposit and dissemination of scientific research documents, whether they are published or not. The documents may come from teaching and research institutions in France or abroad, or from public or private research centers.
L'archive ouverte pluridisciplinaire HAL, est destinée au dépôt et à la diffusion de documents scientifiques de niveau recherche, publiés ou non, émanant des établissements d'enseignement et de recherche français ou étrangers, des laboratoires publics ou privés. 
La préférence pour les comportements prosociaux chez les bébés de 6 mois

Preference for prosocial behaviors in 6-months-old infants

\title{
HOLVOET Claire, SCOLA Céline, ARCISZEWSKI Thomas, \& PICARD Delphine
}

Aix Marseille Université - Centre PsyCLE EA3273

29 avenue Schuman, 13621 Aix-en-Provence, France

\begin{abstract}
Résumé
De nombreuses études montrent des préférences précoces des jeunes enfants pour des agents prosociaux. Cependant, des études récentes ont trouvé des résultats contradictoires quant à l'influence de l'apparence sur ces préférences. La présente étude a pour objectif de déterminer l'influence de l'apparence faciale/l'ethnie sur la préférence des bébés de 6 mois pour des comportements prosociaux. Dans une étude utilisant des mesures oculométriques, nous avons exposé des bébés à des animations vidéos afin de tester leurs préférences: 1) pour un agent prosocial vs. antisocial, 2) pour un agent de leur ethnie vs. d'une ethnie étrangère, et 3) lorsque ces 2 aspects (prosocial $x$ ethnie) sont mis en compétition. Les deux conditions contrôles révèlent une préférence pour l'agent prosocial et l'agent d'une ethnie étrangère. Aucune préférence n'a été identifiée au niveau du groupe dans la troisième condition dans lesquels ces deux éléments sont en concurrence. Cependant, une analyse par profils montre que lorsque cette situation est source de conflit au regard des préférences identifiées dans les conditions contrôles, les bébés expriment une préférence pour l'agent antisocial d'une ethnie étrangère. Ainsi, ils traitent l'apparence de façon prioritaire sur le comportement.
\end{abstract}

Mots clés : Petite enfance ; préférence visuelle ; comportements prosociaux ; ethnie; oculométrie

\begin{abstract}
There is growing evidence that infants display preferences for prosocial agents. However, recent studies have reported conflicting results about the impact of appearance on these preferences. The current study investigated the relative influence of face/race on 6-month-old infants' preference for prosocial behaviour. In a short research study featuring animated cartoons where agents interacted in a ball game, we used eye-tracking methodology to assess infants' preferences 1) for prosocial behaviour, 2) for other-race faces, and 3 ) when the two aspects were pitted against one another. The two control conditions revealed preferences for prosocial agents and for other-race faces. No preference could be found at group level in the third condition, where these two aspects competed with each other. However, a profile analysis revealed that when this situation was a source of conflict in terms of the preferences identified in the control conditions, infants show a preference for the other-race antisocial agent, thereby prioritizing faces over behaviour.
\end{abstract}

Keywords : Infancy; visual preference; prosocial behaviour; race; eye-tracking 


\section{Introduction}

Les préférences précoces des jeunes enfants pour les agents qui adoptent des comportements prosociaux sont au cœur d'un champ de recherche depuis une dizaine d'années (Hamlin, Wynn \& Bloom, 2007 ; cf. pour une revue de littérature, Holvoet, Scola, Arciszewski \& Picard, 2016). Deux études seulement se sont intéressées à l'influence de l'apparence faciale des agents sur ces préférences, et ont rapporté des résultats contradictoires. Une première étude (Burns \& Somerville, 2014) a montré que des jeunes enfants Caucasiens de 15 mois cessent d'exprimer une préférence pour un agent prosocial lorsque son visage montre une ethnie différente de la leur. Dans une seconde étude (Scola, Holvoet, Arciszewski \& Picard, 2015), des enfants Caucasiens de 24 à 36 mois maintiennent leur préférence pour l'agent prosocial malgré des changements dans l'apparence faciale (visage de couleur noire, traits faciaux mélangés). La présente étude a pour objectif d'évaluer l'importance donnée à l'apparence et au comportement dans les évaluations sociales chez des jeunes enfants âgés de 6 mois. Précisément, notre étude cherche à déterminer quelle dimension (comportement versus apparence faciale) est traitée de façon prioritaire chez les bébés.

\section{Méthode}

Dans cette perspective, 20 bébés Caucasiens, âgés de 6 mois, ont été exposés à des animations vidéos dans lesquelles trois agents sociaux interagissent au travers d'un jeu de balle. Trois animations vidéos ont été construites afin de tester leurs préférences dans trois conditions. L'animation 1 (condition contrôle prosocial) permet de tester les préférences pour un agent prosocial vs. antisocial. L'animation 2 (condition contrôle ethnie) permet de tester les préférences pour un agent de son ethnie vs. d'une ethnie étrangère. L'animation 3 (condition combinée prosocial $x$ ethnie) met en compétition un agent prosocial de la même ethnie vs. un agent antisocial d'une autre ethnie. Dans les animations 1 et 3, un personnage central joue à la balle et envoie à tour de rôle la balle aux deux personnages qui l'entourent. Le personnage prosocial, le joueur, renvoie la balle afin que le jeu puisse continuer, tandis que le personnage antisocial, l'anti-joueur, garde la balle avant de l'envoyer dans un arbre ayant pour conséquence d'interrompre le jeu (voir Fig. 1). Dans l'animation 2, les personnages entourant le personnage central et présentant une ethnie caucasienne ou étrangère tournent leur tête à tour de rôle vers le personnage central sans interagir avec lui (voir Fig.1).

Les préférences des jeunes enfants ont été mesurées à l'aide d'un eye-tracker (Tobii X2-60) durant le déroulement de l'animation vidéo (la phase dynamique) et durant une présentation des deux personnages, agrandis et statiques, à la fin de l'animation (la phase test). Des aires d'intérêts ont été définies autour des personnages situés à gauche et à droite du personnage central. Les enfants ont visionné les 3 animations vidéos dans une pièce du BabyLab ${ }^{1}$, installés dans une chaise adaptée positionnée à $60 \mathrm{~cm}$ de l'écran. Les conditions contrôles (animations 1 et 2) ont toujours été présentées avant la condition combinée (animation 3), et dans un ordre contrebalancé au sein des sujets. Le côté des personnages ainsi que la nature du comportement produit en premier ont également été contrebalancés.

Les préférences sont calculées (pour la phase dynamique et pour la phase test) à partir de la formule suivante : temps alloué à la fixation d'un personnage (e.g., prosocial) divisé par le temps passé sur les deux personnages (prosocial + antisocial) ; elles varient de 0 à 1, avec 0.50 représentant une préférence sur le seuil du hasard. Les résultats nous permettront de déterminer quel comportement et quelle ethnie sont préférés par les jeunes enfants de 6 mois, puis d'identifier quelle dimension (comportement versus apparence faciale) est privilégiée dans les évaluations sociales.

\footnotetext{
${ }^{1}$ Le BabyLab se situe à la Maison de la Recherche, Aix-Marseille Université, France
} 

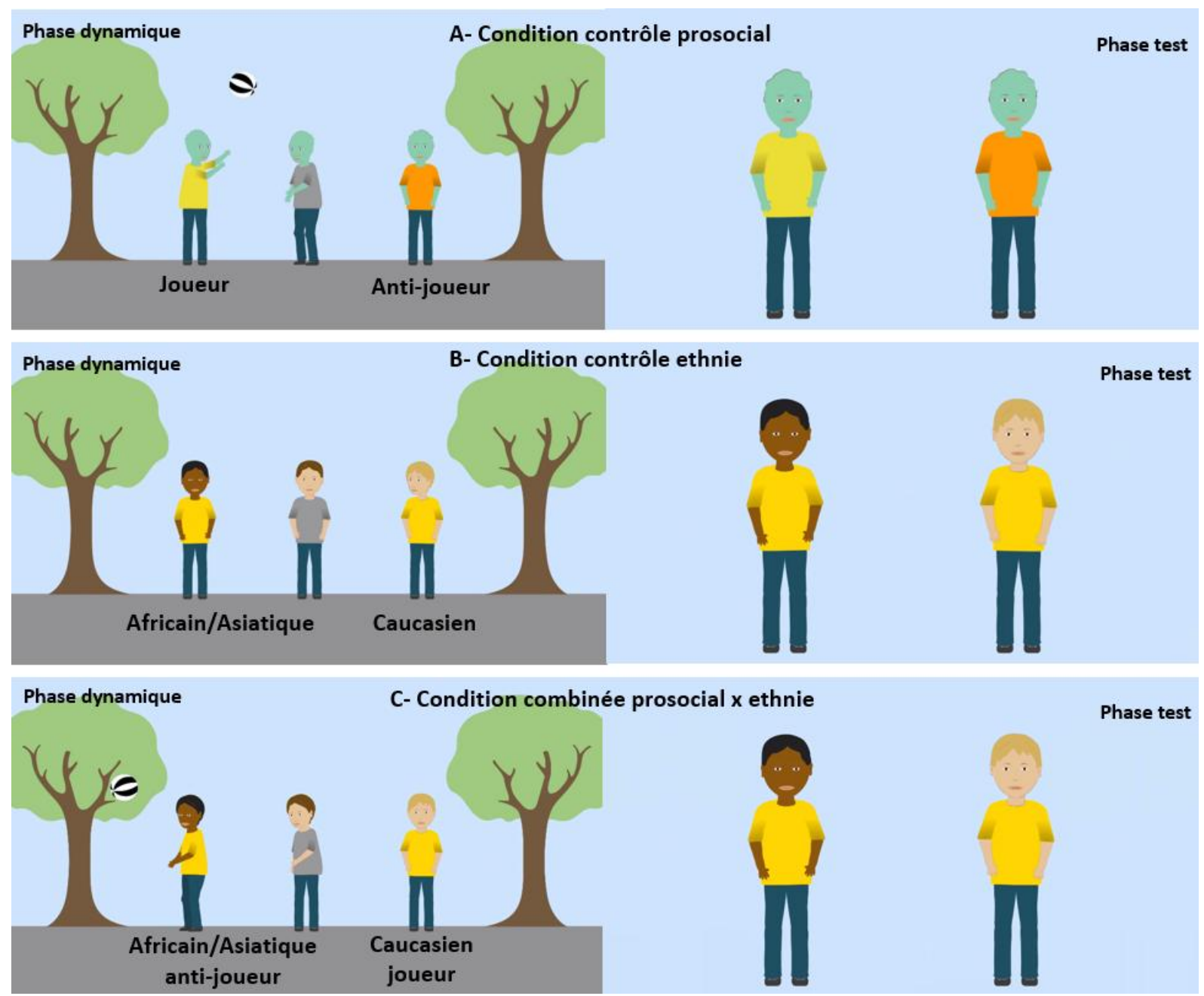

Figure 1. Illustration de chaque condition durant la phase dynamique (scenario) et la phase test. (A) La condition contrôle prosocial présente un personnage central avec une balle qu'il envoie aux personnages situés à sa droite et à sa gauche pour jouer avec eux. Un personnage, le joueur lui renvoie la balle ce qui permet au jeu de se poursuivre, tandis que le second personnage, l'anti-joueur garde la balle et l'envoie dans l'arbre situé à côté de lui. (B) La condition contrôle ethnie présente trois personnages, un situé au milieu de l'écran, et les deux autres situés de chaque côté de ce personnage, qui tournent à tour de rôle leur tête vers lui. L'un de ces personnages présente une ethnie caucasienne, tandis que le second présente une ethnie africaine ou asiatique. (C) La condition combinée prosocial $x$ ethnie présente une combinaison de (A) et (B), dans lequel le personnage central joue avec la balle, l'envoie aux personnages situés à sa droite et à sa gauche pour jouer avec eux. Un personnage, le joueur caucasien, lui renvoie la balle ce qui permet au jeu de se poursuivre, tandis que le second personnage, l'anti-joueur africain ou asiatique garde la balle et l'envoie dans l'arbre situé à côté de lui.

\section{Résultats}

Les résultats montrent une préférence à 6 mois d'une part pour l'agent prosocial durant la phase dynamique (vs. antisocial; $\mathrm{M}=0.54, \mathrm{SD}=0.06 ; t=2.85, p=0.005$ ) et d'autre part pour l'agent d'une ethnie étrangère (vs. de sa propre ethnie) durant la phase dynamique $(\mathrm{M}=0.31, \mathrm{SD}=0.26 ; t=3.24, p$ $=0.002$ ) et durant la phase test $(M=0.39, S D=0.22 ; t=2.32, p=0.01)$. Lorsque ces deux aspects sont mis en compétition (i.e., agent prosocial de la même ethnie vs. agent antisocial d'une autre 
ethnie), aucune préférence n'est observée si l'analyse est menée au niveau du groupe ( $M=0.48, S D=$ $0.08 ; t=1.16, p=0.25 \mathrm{~ns})$. Nous avons réalisé une analyse par profils, c'est-à-dire qui tient en compte les préférences individuelles des bébés dans les conditions contrôles. Ceci nous a amené à distinguer deux types de profils : des profils « neutres » $(n=9$; e.g., pas de préférence pour un comportement ou pour une ethnie) pour lesquels la combinaison prosocial-ethnie familière versus antisocial-ethnie étrangère ne présente pas une situation conflictuelle par rapport aux préférences initiales des bébés et des profils « conflictuels » $(n=11$; e.g., préférence prosocial, et préférence ethnie étrangère) pour lesquels la combinaison prosocial-ethnie familière versus antisocial-ethnie étrangère présente une situation conflictuelle par rapport aux préférences initiales des bébés (i.e., ils vont devoir « choisir » en quelque sorte entre leur attirance pour le prosocial et leur attirance pour l'ethnie étrangère). Cette analyse montre que la répartition des choix dans la condition combinée varie de façon significative en fonction des ces deux types de profils (test exact de Fisher, $p=0.049$ ). Plus précisément, lorsque la situation est "neutre", les bébés préfèrent l'agent prosocial de leur ethnie ( $n=6$ sur 9), mais lorsque la situation est "conflictuelle", ils préfèrent l'agent antisocial d'une ethnie étrangère $(n=8$ sur 11$)$.

\section{Conclusion}

Les résultats montrent que les jeunes enfants de 6 mois préfèrent 1 ) un agent prosocial (à un agent antisocial) et 2) un agent d'une ethnie étrangère (à celui de sa propre ethnie) dans le cadre d'animations vidéos. L'absence de préférence pour un agent prosocial durant la phase test confirme que les mesures réalisées pendant la phase dynamique sont souvent plus sensibles pour détecter les préférences des jeunes enfants, comme montré récemment (Cowell \& Decety, 2015). Enfin, lorsque les bébés sont confrontés à une situation faisant intervenir deux dimensions (i.e., la condition combinée prosocial $x$ ethnie), aucune préférence n'est identifiée au niveau du groupe. Cependant, une analyse par profils démontre que les bébés pour lesquels cette situation est " conflictuelle » (i.e., en considérant les préférences qu'ils ont exprimées pour un agent en raison de son comportement et pour un agent en raison de son apparence) expriment une préférence pour l'agent antisocial d'une ethnie étrangère. Ces résultats suggèrent que pour les bébés de 6 mois l'apparence est traitée de façon prioritaire sur le comportement lorsque ces deux éléments sont en concurrence.

\section{Références}

Burns, M. P., \& Sommerville, J. A. (2014). "I pick you": The impact of fairness and race on infants' selection of social partners. Frontiers in Psychology, 5, 1-10.

http://doi.org/10.3389/fpsyg.2014.00093

Cowell, J. M., \& Decety, J. (2015). Precursors to morality in development as a complex interplay between neural, socioenvironmental, and behavioral facets. Proceedings of the National Academy of Sciences, 112(41), 201508832. http://doi.org/10.1073/pnas.1508832112

Hamlin, J. K., Wynn, K., \& Bloom, P. (2007). Social evaluation by preverbal infants. Nature, 450(7169), 557-559. http://doi.org/10.1038/nature06288

Holvoet, C., Scola, C., Arciszewski, T., \& Picard, D. (2016). Infants' preference for prosocial behaviors: A literature review. Infant Behavior and Development, 45, 125-139.

http://doi.org/10.1016/j.infbeh.2016.10.008

Scola, C., Holvoet, C., Arciszewski, T., \& Picard, D. (2015). Further evidence for infants' preference for prosocial over antisocial behaviors. Infancy, 20(6), 684-692. http://doi.org/10.1111/infa.12095 